\title{
LA INNOVACIÓN EN LA EDUCACIÓN SUPERIOR EN ENFERMERÍA Y LOS APORTES DEL DISEÑO DE INSTRUCCIÓN
}

Alejandrina Arratia Figueroa*

FIGUEROA, A.A. La innovación en la educación superior en enfermería y los aportes del diseño de instrucción. Rev.latino-am.enfermagem, Ribeirão Preto, v. 7, n. 2, p. 5-13, abril 1999.

El presente artículo fundamenta el rol de la educación en enfermería, respecto a asumir una constante innovación y mejoramiento de la calidad de la docencia, considerando las demandas sociales y de nuevos roles profesionales. Plantea como una alternativa los aportes del diseño de instrucción. El cual, ofrece fundamentos educacionales que orientan a la creación de ambientes de aprendizaje propicios, acordes a los requerimientos de la población usuaria y del sistema educativo. El éxito de la innovación propuesta, exige una evaluación continua y una intencionalidad compartida por parte de los docentes para asegurar la consolidación y permanencia frente al cambio.

TÉRMINOS CLAVES: innovación educativa, diseño de instrucción

\section{INTRODUCCIÓN}

La evaluación y control de los sistemas de enseñanza superior en general y en particular los de enfermería, llevan implícitos la apreciación de constantes presiones de variado orden (demandas sociales, económicas y tecnológicas), para cuya atención precisan disponer de métodos educativos, de investigación y evaluativos adecuados, que le permitan mejorar los urgentes requerimientos de actualizar la formación de sus profesionales.

Es así, como la docencia debe transcender el proceso de enseñanza-aprendizaje, favoreciendo una renovación que considere: el avance científico, la versatilidad de los contenidos a enseñar, la incorporación de variadas metodologías que reemplacen la insuficiencia de las clases expositivas, el establecimiento de procesos de educación permanente que abran posibilidades a la educación continua, las expectativas de nuevos roles profesionales, sociales y de interdisciplinariedad, entre otros.

Sin embargo, las reformas en el ámbito educativo superior ofrecen con frecuencia resistencia, incertidumbre, inseguridad, lo que está basado fundamentalmente en la tradición de años y en posturas universitarias rígidas y rutinarias. Esto implica, mantener una constante actitud de alerta, de motivación, de conocimientos y de trabajo en equipo que lleven a superarlas.

De acuerdo a la problemática señalada, el presente artículo plantea la necesidad de innovar en la educación en enfermería, como una forma de optimizar y mejorar en forma amplia y profunda la calidad y efectividad de la misma, utilizando como una alternativa los aportes del diseño de instrucción. Para lograr el propósito, se presentan algunos antecedentes que afectan al sistema universitario en general, los que en particular pueden también ser focalizados a la situación de enfermería. Destacándose de esta forma, el rol activo y responsable que le cabe a la misma, frente al mejoramiento de la enseñanza que imparte.

Se describen a su vez, algunas metodologías educativas y las principales propiedades y características del diseño instruccional.

Cabe destacar que la educación en enfermería, debe ser asumida estimando un trabajo en equipo institucional, que faculte utilizar y desarrollar recursos de aprendizaje concordantes a los requerimientos actuales de la sociedad, aprovechando la actividad científicotecnológica y empleando los medios existentes en toda su potencialidad. Lo que involucra la creación de ambientes propicios al crecimiento individual de los alumnos y al desenvolvimiento académico, favoreciendo una formación profesional activa, creativa $\mathrm{y}$ cuestionadora.

\footnotetext{
* Profesora de la Pontificia Universidad Católica de Chile. Magister en Diseño de Instrucción. Doctoranda del programa, Filosofia em Enfermagem. Universidade Federal de Santa Catarina. Florianópolis/Brasil
} 


\section{INNOVACIÓN EN LA DOCENCIA UNIVERSITARIA}

\section{Problemas actuales de la educación superior latinoamericana}

Las universidades en América Latina han enfrentado en los últimos años, una serie de cambios ocasionados fundamentalmente por el aumento de la demanda de ingresos. Entre los factores determinantes se encuentran por ejemplo: el crecimiento demográfico de la población juvenil, el incremento de la condiciones de vida y la incorporación cada vez más notable de la mujer al trabajo productivo y a la educación superior. A su vez, circunstancias económicas, sociales y culturales de los diversos países, han favorecido un acelerado avance científico y tecnológico, involucrando mayores condiciones de competencias tanto a nivel laboral como en la renovación del conocimiento.

Por otra parte, los diferentes movimientos sociales que condicionan las actividades de enseñanza, las diferentes características de los grupos de alumnos, la falta de formación pedagógica y las diferentes concepciones tanto ideológicas, pedagógicas como políticas de los docentes; son factores que comprometen el grado de acuerdo y de compromiso de los profesionales en relación a los objetivos propuestos, produciendo una falta de sintonía y alterando el éxito de la vida académica.

Según BRUNNER (1993), los debates en América Latina, en lo concerniente a la pedagogía universitaria han establecido reorganizaciones profundas y severas readaptaciones, en el intento de responder a las imposiciones del medio social. El autor, realiza una tentativa de diagnóstico acerca de lo señalado, identificando diferentes aspectos, entre los cuales se encuentran:

* La existencia de un notorio cambio en la actitud de los Gobiernos frente a la educación superior.

* La expansión de la enseñanza superior llevada a efecto, en la mayoría de los casos, sin una adecuada regulación pública.

* La percepción de un deterioro de la calidad de la enseñanza superior, en general.

* La presencia de problemas referentes a la calidad y equidad, las que han aumentado la falta de eficiencia de los sistemas de educación superior.

Surge así, el requisito de acreditar y evaluar debidamente la actividad institucional, para "restaurar un sentido de seriedad y eficacia en la educación superior" (SIMONDS \& RIOS, 1993, p.106). Esto propone, la implementación de procesos evaluativos apropiados, actualizados y pertinentes, junto a un homogéneo compromiso de todos los profesionales, respecto a la autoevaluación de la entidad universitaria. Según los autores, la proposición de acreditación emerge de acuerdo a diversos factores, como por ejemplo: falta de claridad y precisión frente a la misión y objetivos particulares; desproporción entre recursos y demanda; discordancia entre la rápida acumulación de información científica y la lentitud de los cambios curriculares y la falta de atención sistemática, que permita emitir un juicio sobre la efectividad, eficiencia y productividad.

\section{La innovación en la educación superior}

Las universidades, son las responsables de ofrecer metodologías acordes al avance científico, tecnológico y a los requerimientos de los alumnos y por otra parte, promover el desarrollo de investigaciones. Se establece así, la imperante condición de innovar la actividad docente desde una perspectiva realista, activa y comprometida, realizando esfuerzos por conducir sistemas educativos de calidad, garantizando posibilidades que permitan llevar a cabo, investigaciones en forma oportuna. Esto sugiere no solamente el perfeccionamiento del profesor, sino que además, obliga abarcar un conjunto de acciones en forma amplia con el propósito de dar soluciones y provocar mudanzas tanto a nivel institucional como de actitudes de todos los participantes del proceso enseñanza-aprendizaje.

La sociedad plantea hoy en día a los centros de formación superior, el reto de un mundo cada vez mas complejo, el que se distingue por la rápida expansión del conocimiento, situación que genera transformaciones permanentes que afectan la existencia en todos sus ámbitos. Enfrentar el futuro con creatividad, es hoy la aspiración máxima de todo sistema educativo. Por ello, se ha iniciado un proceso de análisis, que propone repensar el modelo tradicional (SOLAR, 1993).

La enseñanza universitaria en el ámbito de la salud, enfrenta por su parte, un propio fenómeno de recombinación de conocimientos, como consecuencia de la rapidez con que estos se producen. "La velocidad de acumulación de conocimientos es mucho mayor que la velocidad a la cual se evalúa el impacto de cada hallazgo en otros campos de la ciencia" (LAGE, 1995, p.248).

Lo anterior reafirma el hecho, que la función académica no puede reducirse sólo a la trasmisión de conocimientos a través de clases expositivas, sino que ella, debe garantizar el "acceso y desarrollo a expresiones creativas y de aprendizajes esenciales, con una enseñanza capaz de personalizarse frente a la diversidad de capacidades, intereses, motivaciones y expresiones culturales" (RODRIGUEZ, 1994, p.9).

Los avances especialmente en relación a la potencialidad de la tecnología educativa, del diseño de instrucción y de la creciente incorporación de medios alternativos para hacer frente a los desafíos, hizo que a 
partir de 1970, las entidades educacionales tomaran conciencia y se interesaran por concebir programas de capacitación en pedagogía, creándose programas sistemáticos de perfeccionamiento a los profesores.

Sin embargo, la propuesta anterior no ha presentado resultados adecuados, dado que se fortaleció la investigación en desmedro de la docencia, al respecto OLIVAREZ \& ZAVALZA (1987, p.5), comentan que "es deber de la universidad propender a la excelencia académica y para ello es fundamental alcanzar una enseñanza efectiva, la universidad debe preocuparse no sólo del perfeccionamiento de los docentes en el campo de la disciplina específica en que imparten su docencia, sino además en el campo pedagógico".

Por otra parte, en el desarrollo de la investigación, se observan en general deficiencias que determinan que el proceso educativo se oriente sólo a lo establecido en los currículos, no dejando la posibilidad a la búsqueda investigativa como elemento de formación del egresado. Dicho profesional "se dedicará a aplicar las soluciones que le fueron entregadas descontextualizadas. En pocas ocasiones intentará averiguar si es posible hacer las cosas de otra manera o encontrar explicaciones alternas" (DE MIGUEL, 1991, p.33).

Se requiere por tanto, de innovaciones que incidan en el progreso y motiven la investigación. Incentivando a través de esta última, la búsqueda de variables inherentes al proceso educacional, con el propósito de obtener resultados en aspectos, tales como: rendimiento y actitudes de los estudiantes, estilos cognitivos, estrategias de enseñanza, entre otros; los que entregarán información pertinente respecto a la calidad de los medios instruccionales y de la enseñanza (RODRIGUEZ \& VERGARA, 1993).

Cabe sin embargo destacar, que los cambios en la enseñanza superior se ven afectados por rutinas ya establecidas y por la tradición acuñada por tantos años en los estilos catedráticos (GONZÁLEZ,1993). Sumándose a esto, una cierta resistencia, incertidumbre e inseguridad frente a los logros y a fracasos anteriores. Esto alerta a mantener una constante actitud de motivación, de conocimientos y de trabajo en equipo, que capaciten a los profesionales a superar dichas tendencias negativas y a comprometerse con la innovación.

\section{La educación en enfermería y su imperante necesidad de innovar}

Los cambios ocurridos en el área de la tecnología, de la biotecnología, en el intercambio de bienes y servicios y en la educación y salud de la población, exigen la formación de recursos humanos capacitados, para replicar y manejar dicha diversidad y para entender la complejidad de los factores que ejercen influencia en la promoción y en el mantenimiento de la salud de la población (MIOTTO \& GARZÓN, 1995).

Lo anteriormente señalado, junto a la problemática que afecta a las universidades en general, desafía a las escuelas de enfermería a innovar, planteándose la misión de trabajar en pro de la calidad de su enseñanza. Reaccionando favorablemente, frente a la diversidad de condiciones que emergen de la sociedad, de los servicios de salud y de la comunidad; desarrollando mecanismos que articulen adecuadamente, educación, atención en salud e investigación en enfermería.

Para lograr tal propósito, precisan replantearse los planes de estudio, estimando que "los cambios curriculares existentes han sido parciales y simplistas, no produciendo las transformaciones profundas que son necesarias. Esto es debido en parte, al hecho de que los docentes tienen preparación insuficiente, o no tienen la experiencia docente requerida" (KISIL, 1993, p.39).

Se espera entonces, la implementación de currículos innovativos que promuevan una educación crítica, deliberada y participativa. Esto involucra, la redefinición de roles del docente, del alumno y de la organización curricular, favoreciendo un clima organizacional creativo el cual incorpore asimismo una renovada y dinámica concepción de evaluación. Permitiendo la formación de profesionales activos y creativos, capaces de enfrentar situaciones nuevas, de resolver problemas y de adaptarse a un mundo cambiante; el cual tiene como expectativa, una atención en salud que refleje un conocimiento actualizado y una actitud de alerta y disposición frente a la educación permanente.

Para responder a las deficiencias detectadas, se plantea que enfermería tome un papel activo al respecto, incorporando en su función educativa, un diseño instruccional que conduzca a encontrar el camino mas conveniente. Esto implica mantener un desarrollo armónico, entre investigación y docencia, con el objeto de promover la modernización y renovación de la enseñanza de la profesión. Proyectándose al futuro de una forma amplia y ofreciendo alternativas que faciliten entre otros, una creciente formación de post-grado.

Innovar en la educación en enfermería, implica por tanto, la demostración de un rol pedagógico que acredite y asegure un proceso que respete actitudes, valores, competencias, habilidades y destrezas. El diseño de métodos y estrategias de enseñanza debe atender a las formas individuales de aprender y favorecer el desenvolvimiento de un buen pensamiento en el alumno; el que se espera, sea crítico y creativo, motivando un aprendizaje continuo, para que logre ser autónomo, con una mentalidad cuestionadora y familiarizado con el proceso de aprender.

El concepto de aprendizaje activo, toma una 
especial vigencia desde la perspectiva de una educación innovativa, "cuando son reunidos los esfuerzos intelectuales tanto de estudiantes como de profesores. El aprendizaje activo, es necesario para el desempeño del pensamiento crítico, es lo que identifica a una persona educada de una meramente entrenada" (ANGELO,1994, p.12).

Los docentes enfermeros, no pueden pretender que los alumnos, aprendan a resolver instancias conflictivas de la práctica sólo a través de la observación del profesor, por el contrario, ellos deben ser expuestos a procedimientos de laboratorio en dónde puedan discutir acerca de posibles soluciones, para luego tomar las decisiones mas acertadas. Estas vivencias, se tornan especialmente importantes considerando que el futuro profesional, se enfrentará en el día a día de su quehacer a complejos y cambiantes dilemas, dentro de un contexto que involucra tanto sus propios intereses, como las peculiaridades históricas, sociales y políticas del medio en que trabaja.

Por último es posible señalar, que una docencia eficaz estará delimitada en gran medida, por el trabajo en equipo entre académicos, por el interés en el perfeccionamiento docente, por la dedicación y competencia de cada uno de ellos y por el constante apoyo de las respectivas autoridades universitarias. Evaluando oportunamente el impacto de los cambios curriculares, a través del seguimiento oportuno de los egresados, con el propósito de obtener información, en relación a los logros obtenidos, en la forma mas oportuna posible.

\section{Opciones innovativas en la educación en enfermería}

Para favorecer la efectividad del proceso educativo existen diversas opciones metodológicas y recursos, que se pueden seleccionar de acuerdo al diseño de instrucción realizado; respondiendo a lo requerido por la población destinataria, a las instancias educativas y a los medios institucionales disponibles. A continuación se mencionan algunas alternativas al respecto y se describen sus principales atributos.

\section{Metodológicas:}

* Modalidad centrada en problemas: corresponde a una estrategia que trabaja en la perspectiva de la educación libertadora. Parte de la base, de que en un mundo de cambios rápidos, lo importante es el aumento de la capacidad del alumno para detectar los problemas reales y buscarles solución original y creativa. Freire, es el gran impulsador de esta metodología. El educador propone una práctica educativa, orientada a promover y desarrollar en los estudiantes, una conciencia crítica, dialógica, comunicativa, respetuosa de las características propias de cada uno de ellos y dirigida a fomentar una participación activa, autónoma y auténtica.

La praxis de Freire, fortalece una ideología humanizadora y esencialmente respetuosa de la libertad de las personas; este aspecto es muy importante de ser trasmitido y vivenciado por los educandos, dado que encierra una dimensión ética, que se desea prevalezca y se refleje posteriormente en el desempeño profesional (FREIRE, 1986).

Una docencia innovativa, debe plantearse la constante preocupación de considerar la individualidad de los alumnos y su derecho a participar activamente en el proceso educativo. En la medida que las vivencias de los mismos sean positivas y renovadoras, se estará contribuyendo a estimular una actitud de respeto y autenticidad en su posterior actuación profesional.

* Modalidad centrada en el enfoque del enseñar a pensar: es una metodología que enfatiza en las habilidades del pensamiento, propiciando el desarrollo de una postura crítica y creativa, la que requiere de una enseñanza que estimule el autoexamen del pensamiento en términos de claridad, consistencia, sustantividad, contexto, dialoguicidad y prioridad. "El compromiso activo de los alumnos constituye un aspecto importante de esta enseñanza" (NICKERSON et al., 1994, p.12).

De acuerdo a los autores señalados, enseñar a pensar implica considerar: las capacidades subyacentes al pensamiento, los métodos que ayudan al pensamiento, los conocimientos sobre el pensamiento y las actitudes que conducen al pensamiento. Esto implica reorganizar los planes de estudio, en torno a las habilidades específicas del mismo y no en relación a los contenidos, enfatizando en especial en aquellas que intervienen en actividades de orden superior, tales como el razonamiento, el pensamiento creativo y la solución de problemas.

SOLAR (1993), por otra parte, plantea que para estimular el desarrollo del pensamiento creativo el docente precisa utilizar diversas técnicas a través de talleres en distintas áreas, entre las que se pueden mencionar: el pensamiento visual, el pensamiento metafórico, la fantasía, la lectura creativa, el arte de preguntar, la lluvia de ideas, el análisis morfológico, la síntesis creativa, el método delfos y la solución de problemas entre otros.

* Modalidad no presencial: modalidad educativa que considera la aplicación de un sistema de instrucción, que implica nuevos roles tanto para alumnos como para docentes, nuevas actitudes y nuevos enfoques metodológicos. Esta metodología genera nuevas estrategias en cuanto a la combinación de recursos, medios y formas de trabajo. Precisando para el logro de metas, un trabajo colaborativo, consistente y coordinado entre las diferentes personas que conforman la entidad educativa (WILLIS, 1993).

Según HOLMBERG (1987), la educación no 
presencial, comprende las diversas formas de estudio a todos los niveles, que no estén bajo la supervisión continua e inmediata de docentes o tutores presenciales con sus alumnos en aula, pero no obstante, se benefician de la planificación, guía y enseñanza tutorial. La introducción de esta modalidad en las universidades tradicionales, constituye un avance significativo y se ha adoptado en varias instituciones Latinoamericanas.

En la educación a distancia, la exactitud de la extensión y la naturaleza de la estructura de la comunicación, variarán de acuerdo al tipo de medio, del contenido a enseñar y de la población destinataria; la producción de programas de este tipo requieren siempre de una elaboración más costosa y de mayor consumo de tiempo (MOORE, 1987).

* Modalidad semipresencial o mixta: surge como respuesta a la necesidad de alternar la educación presencial y la no presencial, en la búsqueda de modos que favorezcan optimizar el quehacer universitario tradicional. Se presenta como una forma de complementariedad y mejoramiento al quehacer docente, permitiendo al alumno, adecuar el estudio a su estilo personal. BOTIJA (1988), destaca a las tutorías, como el componente esencial de un sistema semipresencial, pues a través de ellas, se lleva a cabo en gran parte el proceso de retroalimentación académica y pedagógica, facilitando y manteniendo en todo momento la motivación de los estudiantes y apoyando el proceso de enseñanzaaprendizaje.

\section{Recursos de aprendizaje}

* Audiovisuales: amplían la capacidad comunicativa en la docencia, por medio de videos, diaporamas, audioramas, set de diapositivas o una combinación entre ellos.

* Multimedios: enriquecen y dinamizan los procesos de enseñanza-aprendizaje. Están compuestos por una serie de medios instruccionales, los que utilizados en su conjunto, permiten al alumno acrecentar, complementar, potenciar y reforzar la adquisición y/o práctica de un nuevo conocimiento.

* Computador: representa un medio de creciente sofisticación para ser usado desde aprendizajes simples hasta la elaboración de programas heurísticos de alta complejidad (programas tutoriales, softwares educacionales). Permite un aprendizaje interactivo e individual a cada alumno, sirviendo de apoyo o complemento a las clases, como material de ejercitación, de evaluación o profundización.

La implementación y utilización de diversos medios instruccionales en la docencia, propone a su vez, la creación de centros de recursos de aprendizaje. Los que llevan a satisfacer instancias educativas de una variada población (estudiantes de enfermería, equipo de salud, usuarios de la salud y/o comunidad en general). Se pueden definir: como el lugar donde se almacena la mas amplia gama de información, en múltiples y diversas formas (modelos, material escrito, medios audiovisuales y/o computacionales), a fin de ponerlas al alcance de todos los participantes de la instrucción.

Según CABERO (1985), cumplen con un variado número de funciones, entre ellas las mas importantes son: de préstamo y producción de material audiovisual, de control y mantenimiento, de perfeccionamiento docente y de otros profesionales de la salud, de evaluación del material producido y de las actividades del centro, de documentación e información, de elaboración de diseños de instrucción y de difusión de experiencias realizadas.

\section{El diseño de instrucción y la educación superior en enfermería}

\section{Conceptualización}

REIGELUTH (1983), conceptualiza el diseño de instrucción, como una disciplina interesada en la comprensión y el mejoramiento de un aspecto de la educación, el proceso de instrucción. Como área de estudio, la relaciona a la búsqueda de formas y métodos "de como enseñar". El autor la considera una ciencia de enlace, es decir, un cuerpo de conocimientos que prescribe acciones instruccionales, para optimizar los resultados de dichas acciones. Por tanto, su interés básico como disciplina, está centrado en la prescripción de métodos óptimos de instrucción, para originar cambios en el conocimiento y destrezas del estudiante.

RITCHEY (1986), se refiere al diseño de instrucción como una ciencia creativa, una disciplina en sí misma, una área metodológica de estudio y práctica con base en la investigación empírica. Como tal, establece especificaciones detalladas para el desarrollo, evaluación y mantención de situaciones que faciliten el aprendizaje de grandes y pequeñas unidades. Abarca un amplio rango de actividades, incluyendo los pasos de la planificación inicial. Opera a través de la creación de procedimientos que aseguran la aplicación continua de los procesos instruccionales.

Según KEMP (1985); POSNER \& RUDNITSKY (1986); GAGNÉ et al.(1988); el diseño de instrucción puede ser definido, como el arte de crear instrucción clara y efectiva, a través de un proceso sistemático, que presenta un completo análisis de necesidades de aprendizaje, metas y desarrollo de un sistema de entrega, tomando en cuenta las singularidades de la población usuaria. Incluye el desarrollo de materiales, actividades y métodos instruccionales y el ensayo y revisión de toda la instrucción, junto a las respectivas actividades de evaluación. 
DICK \& CAREY (1989), por su parte, concuerdan con los aspectos de las conceptualizaciones anteriores y enfatizan en que el proceso instruccional puede por sí mismo, ser visto como un sistema, en el que cada componente es crucial para lograr un aprendizaje exitoso. El propósito del sistema es originar aprendizaje; sus componentes están formados por: alumnos, docentes, materiales instruccionales y ambientes de aprendizaje.Todos ellos, desenvuelven un rol esencial e interactúan con el fin de alcanzar la meta instruccional propuesta, en forma efectiva, significativa e interactiva. Los autores, formulan un modelo de diseño de instrucción, el que ilustra el procedimiento general conducente a la planificación, desarrollo y evaluación del proceso de enseñanza-aprendizaje.

Los componentes del sistema según Dick y Carey, corresponden: a la meta de instrucción, alumnos, objetivos, test basados en criterios, estrategia de instrucción, materiales de instrucción, evaluación formativa y sumativa.

WILLIS (1993), conceptualiza a su vez el diseño de instrucción, como un proceso sistemático, que permite desarrollar instrucción. El proceso como tal, provee un camino que favorece reanalizar y revisar los contenidos de cursos o programas, basados en las características y requerimientos de los alumnos. Esto es esencial, especialmente en un sistema de entrega de instrucción a distancia, para que éste logre ser efectivo.

En la conceptualización anterior, se enfatiza en especial, en el diseño de instrucción como proceso de desarrollo, mantención y corrección de sistemas a distancia. Dicha modalidad, considera la aplicación de una instrucción que implica nuevos roles, nuevas actitudes y nuevos enfoques metodológicos, generando nuevas estrategias en cuanto a la combinación de recursos, medios y formas. Requiere para el logro de metas, un trabajo colaborativo, consistente y coordinado. Mientras cada persona juega un rol diferente, tiene una meta en común: proporcionar una experiencia de aprendizaje a distancia relevante para un grupo seleccionado de alumnos (WILLIS, 1993; SAUVÉ, 1993).

\section{Ambitos de aplicación, propósitos y aportes del Diseño de Instrucción}

En cuanto al ámbito de aplicación, el diseño de instrucción es aplicable a una variedad de contextos educativos. En el área educacional en enfermería, estos pueden corresponder a: la enseñanza de pre-grado y/o post-grado, la educación de adultos (usuarios de la salud, personal auxiliar y otros profesionales de la salud), sistemas educativos a distancia, sistemas semipresenciales, la educación permanente en los servicios de salud, situaciones de aprendizaje comunitarios, sistemas educativos formales o informales y otros.

En relación a sus propósitos, el diseño de instrucción como actividad profesional, orienta a decidir que métodos de instrucción son mejores para originar cambios deseados en el conocimiento y destreza de los alumnos; esto implica, comprender, mejorar y aplicar medios instruccionales, que faciliten y apoyen el aprendizaje. Esta intención es propia de la enseñanza donde quiera que ella ocurra, ya sea, entre un tutor y un estudiante, en una sala de clases, grupos de adultos, o en un lugar de trabajo.

Como disciplina, su propósito es producir conocimientos acerca de modelos instruccionales óptimos, utilizando para ello, aportes de teorías psicoeducacionales, investigación y áreas formales de pesquisa y práctica.

El apoyo de la instrucción al aprendizaje, es algo planeado, que va mas allá del azahar. Como resultado se obtiene un modelo, un plano, de como debería ser la instrucción. De ahí, que el énfasis se coloca principalmente en la planificación, la que debe ser adecuada y oportuna, aspecto que va a permitir a cada alumno aproximarse al máximo a las metas, al empleo óptimo de sus capacidades, y a disfrutar de su vida, interactuando con su medio físico y social, en su o sus propias direcciones. El diseño de instrucción se presenta entonces, como una opción, para rediseñar un proceso educativo en marcha que presente deficiencias, o para dar inicio a uno nuevo, de acuerdo a los requerimientos educativos identificados.

De acuerdo a las diferentes conceptualizaciones señaladas por los autores, al ámbito de aplicación y a los propósitos, del diseño de instrucción se destacan a continuación sus principales contribuciones para la educación en enfermería:

* Su sistematicidad, favorece establecer un camino para examinar problemas y deficiencias instruccionales, planteando procedimientos para resolverlos y para evaluar sus resultados, considerando entre sus supuestos: el tipo de aprendizaje y la instrucción efectiva. Guía a un ordenamiento a través de planificaciones apropiadas, las cuáles hacen posible anticipar e identificar condiciones de enseñanza-aprendizaje, que respondan con métodos óptimos de instrucción al logro de nuevos conocimientos, competencias, habilidades y destrezas, estimulando una aproximación entre teoría y práctica. Permite la creación e instauración de ambientes de aprendizaje para enfermería, que aseguren el desenvolvimiento de un proceso que tome en cuenta actitudes, valores, ritmo de aprendizaje y aspectos afectivos de los alumnos.

* Su dinamicidad, estimula al docente a seleccionar de entre una variedad de posibilidades, la actividad o estrategia educativa mas adecuada respecto: al 
aprendizaje, instrucción y diseño requerido, para alcanzar determinada meta, favoreciendo el enlace entre teorías educativas y práctica.

* Su constante desenvolvimiento y desarrollo, como área metodológica de estudio y práctica, favorece la promoción de la creatividad, del planteamiento de situaciones problematizadoras y del fortalecimiento de estilos cognitivos. Beneficiando de esta forma, el desarrollo de un pensamiento crítico y creativo, estimulando al máximo la utilización de las capacidades de los estudiantes. Fomenta así, la implementación de métodos atractivos y motivadores, acordes al proceso de enseñanza-aprendizaje en el ámbito de la enfermería, tomando en cuenta la multiplicidad de situaciones y ambientes que los alumnos deben enfrentar en su formación.

Proporciona alternativas de diseño, para sistemas educativos a distancia y semipresenciales, los que pueden ser instaurados en programas de post-grado.

* Su orientación hacia el desarrollo, evaluación y mantención de condiciones que faciliten la enseñanza, exigen un análisis completo de necesidades de aprendizaje y metas educacionales, que lleven a desenvolver materiales y actividades instruccionales y a reevaluar el material existente, de acuerdo a las metas planteadas y a las características de la población usuaria.

* Su constante énfasis en las diferentes instancias evaluativas (diagnóstica, formativa y sumativa), garantiza la adecuación y pertinencia del diseño instruccional utilizado. Esto privilegia la corrección inmediata y oportuna de materiales, métodos u otros componentes, gracias especialmente a la evaluación formativa, presente a lo largo de todo el proceso, propiciando una retroalimentación continua.

La educación en enfermería, debe buscar caminos en pro de enfrentar en forma satisfactoria los desafíos. El trabajo multidisciplinar y en este caso, la posibilidad de formar equipos con las facultades de educación, mantienen abierta la probabilidad de mejorar los procesos educativos y de enriquecer el desempeño profesional. La asesoría de educadores que participen en la conducción de diseños instruccionales, de evaluación y/o curricular, o la formación de post-grado, de enfermeras en el área, beneficiará la conducción, optimización y actualización de la enseñanza de la disciplina.

Un diseño instruccional apropiado y pertinente en la docencia en enfermería, constituye un constante estímulo frente a la innovación, favoreciendo el desarrollo y difusión de futuros proyectos institucionales que fomenten a su vez, la realización de concursos de investigación, en el ámbito de la educación superior en enfermería. Las ventajas y/o limitaciones del diseño instruccional, por su parte, precisan ser investigadas, ofreciendo al respecto, una interesante área de pesquisa.

\section{CONSIDERACIONES FINALES}

La educación en enfermería, constituye la base esencial para edificar el desarrollo y progreso de la profesión. Su desenvolvimiento, actualización e innovación, reclama de transformaciones intensas que den respuesta al progreso y mejoramiento de programas de formación y de perfeccionamiento, tanto de pre-grado, post-grado, como de programas, dirigidos al equipo de salud, o a usuarios.

La calidad de la docencia en enfermería, debe garantizar la preparación de profesionales competentes que se identifiquen con su quehacer y que estén alertas a reaccionar al medio social y a las exigencias de los servicios de salud. El crecimiento de la disciplina, implica buscar caminos en pro de enfrentar en forma satisfactoria los múltiples desafíos que se plantean en sus diferentes ámbitos, especialmente en el área de la educación.

La innovación en la docencia en enfermería, es una demanda inmediata que determina una participación activa, creativa y de una postura renovadora junto a un persistente compromiso del equipo docente. El éxito de esta misión, precisa de una intencionalidad compartida y explícita de parte de los profesionales de la institución universitaria, que permita asegurar la consolidación y permanencia al cambio.

Para concluir es posible destacar que la actividad docente en enfermería diseñada sistemáticamente, constituye un rol motivador y de guía, del proceso enseñanza-aprendizaje. El diseño de instrucción, ofrece fundamentos educacionales, que orientan a la generación de ambientes propicios tanto para el crecimiento personal de los alumnos, como para el desarrollo académico. Estimula la práctica, la capacitación y la actualización del conocimiento, con recursos de aprendizaje atractivos, a través de la creación de medios instruccionales acordes a los requerimientos de la población usuaria y del sistema educativo.

Finalmente cabe señalar que la determinación de la eficacia de la educación superior en enfermería, establece la necesidad de planificar e implementar procesos evaluativos y de autoevaluación adecuados, actualizados y pertinentes, que den cuenta en forma oportuna, de las fortalezas y debilidades de las acciones educativas, para realizar las modificaciones y/o correcciones necesarias. 


\section{INNOVATIONS IN NURSING EDUCATION AND THEIR CONTRIBUTION TO THE TEACHING MODEL}

The role of nursing is studied, as it is related to constant innovation and improvement of teaching quality, social requirements and new professional roles. As an alternative, the contribution given by the teaching model is considered. Such model offers educational bases on leisure activities about adequate learning environments, user population requirements and the actual educational system. Favourable results of the proposed innovation will need continuous evaluation shared by the faculty as a means to guarantee the consolidation of this exchange program.

KEY WORDS: educational innovation, instructional model

\section{REFERENCIAS BIBLIOGRÁFICAS}

01. ANGELO, M. Educação em Enfermagem. Rev.Esc.Enfermagem USP, São Paulo, v.28, n.1, p.11-14, abr.1994.

02. BOTIJA, B. El profesor tutor, un profesional al servicio de la enseñanza a distancia. A distancia. Rev. Universidad Nacional a Distancia, España, v.1, p. 7-8, 1988.

03. BRUNNER, J.J. Evaluación y financiamiento de la educación superior en América Latina. Bases para un nuevo contrato social In: Acreditación universitaria en América Latina. Programa políticas y gestión universitaria-CINDA Santiago-Chile, 1993. p.71-90.

04. CABERO, J. Funciones del centro de recursos. Rev.Patio Abierto, n.13, p.2-5, marzo/abril 1985.

05. DE MIGUEL, J.A. Función y características del ciclo básico en la universidad de América Latina en: Docencia universitaria en América Latina.Programa políticas y gestión universitaria. Santiago-Chile: CINDA, 1991. p.21-37.

06. DICK, E.; CAREY, L.The sistematic design of instruction. 3.ed. Glenview, Illinois: Scott, Foresman, 1989.

07. FREIRE, P. Educação o como prática da liberdade. 17.ed. Rio de Janeiro: Editora Paz e Terra, 1986.

08. GAGNE, R.; BRIGGS, L.;WAGER, W. Principles of instruccional design. 3.ed. New York: Holt, Rine hart y Winston, 1988.

09. GONZALES, L.E. Innovación en la educación universitaria en América Latina In: Innovación en la educación universitaria en América Latina. Santiago-Chile: Centro Interuniversitario de Desarrollo-CINDA, 1993. p.21-39.

10. HOLMBERG, B. Status and trends of distance education. 2.ed. Distance Educationa/Revue de l'enseignement à distance, v.2, n.1, Spring/ Printemps, 1987.

11. KEMP, J.E. The instruccional design process. New York: Harper and Row, 1985.
12. KISIL, M. O desenvolvimento da enfermagem na América Latina: uma visión estratégica. Rev.Latino-Am.Enfernagem, Ribeirão Preto, v.1, n.1, p.37-41, janeiro 1993.

13. LAGE, A. Los desafíos del desarrollo: la actividad científica como eje de la formación del personal de salud.Educ.Med.Salud, v.29, n.3-4, p.243256, 1995.

14. MIOTTO, M.DA G.; GARZON, N. Estudio de los programas de especialización y maestría en enfermería en América Latina. Educ.Med.Salud, v.29, n.3-4, p.331-343, 1995.

15. MOORE, M. University distance education of adults. Teach Trends, v.32, n.3, p.13-18, Sept. 1987.

16. NICKERSON, R.; PERKINS, D.; SMITH, E. Enseñar a pensar. 3.ed. Barcelona: Paidós Ibérica, 1994.

17. OLIVAREZ, M.A.; ZABALZA, J. Delineamiento de proyectos evaluativos $y$ análisis de datos. Santiago: Pontificia Universidad Católica de Chile, 1987.

18. POSNER, G.; RUDNITSKY, A. Course design: a guide to curriculum developmen for teachers. 3.ed. New York: Longman, 1986.

19. REIGELUTH, C.H. Instructional design: whats is it? and why is it?. In REIGELUTH, C.H. (ed.). Instructional design theories and models: an overview of their current status. New Jersey: Lawrence Earlbaum, 1983. p.3-36.

20. RITCHEY, R. The theorical and conceptual bases of instruccional design. London:Kogan Page, 1986.

21. RODRIGUEZ, E. Construcción y reconstrucción de la enseñanza y el aprendizaje. Rev. Pensamiento Educativo, Chile, v.15, p.9-10, dic.1994.

22. RODRIGUEZ, E.; VERGARA, A. Innovación y nuevas metodologías en la docencia de la Pontificia Universidad Católica de Chile In: Innovación en la educación universitaria en América Latina. Santiago-Chile: Centro Interuniversitario de Desarrollo-CINDA, 1993. p.171-189. 
23. SAUVE, L. What's behind the development of a course on the concept of distance education? In: KEEGAN,D. (ed). Theoretical principles of distance education. New York, 1993.

24. SIMMONS, H.; RIOS, E. La acreditación en los Estados Unidos In: Acreditación universitaria In América Latina. Programa políticas y gestión. Santiago-Chile: Centro Inteuniversitario de Desarrollo-CINDA, 1993. p.105-107.
25. SOLAR, M.I. Innovación y creatividad en la educación universitaria In: Innovación en la educación universitaria en América Latina. Santiago-Chile: Centro Interuniversitario de Desarrollo-CINDA, 1993. p.151-157.

26. WILLIS, B. Distance education: a practical guide. New Jersey: Englewood Cliffs, 1993. 